\title{
Pediatric mitral valve reconstruction in acute endocarditis
}

\author{
Jacek Juściński ${ }^{1,2}$, Konrad Paczkowski ${ }^{1}$, Maciej Chojnicki ${ }^{1}$, Mariusz Steffens ${ }^{1}$, Anna Romanowicz ${ }^{1}$,
} Katarzyna Gierat-Haponiuk ${ }^{3}$, Wiktor Szymanowicz ${ }^{1}$, Marta Paśko-Majewska ${ }^{1}$, Ireneusz Haponiuk ${ }^{1,3}$

${ }^{1}$ Department of Pediatric Cardiac Surgery, St. Adalbertus Hospital, Gdansk-Zaspa, Poland

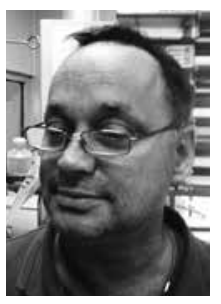

${ }^{2}$ Department of Cardiac Surgery, Medical University of Gdansk, Gdansk, Poland

${ }^{3}$ Chair of Physiotherapy, Faculty of Rehabilitation and Kinesiology, Gdansk University of Physical Education and Sport,

Gdansk, Poland

Kardiochirurgia i Torakochirurgia Polska 2018; 15 (2): 143-145

Although acute endocarditis is still a rare disease in the pediatric population, heart valve repairs in children have become procedures of choice, especially in emergency settings. We present a case of an 11-year-old patient with acute endocarditis and subsequent valve incompetence after infective destruction, who underwent extensive mitral valve (MV) repair with an individually designed surgical technique.

The 11-year-old boy was admitted to the Department of Pediatric Cardiac Surgery because of heart failure presenting with hectic temperature up to $40^{\circ} \mathrm{C}$ and clinical signs of sepsis with concomitant MV incompetence. The boy was suffering from shortness of breath and dyspnea during exercise with NYHA class II/III. Bacteriological analysis showed Staphylococcus aureus cultures obtained from blood and a healing wound on the leg. Antibiotic treatment was started upon an antibiogram. Due to clinical signs of endocarditis, transthoracic echocardiography (TTE) was performed, showing an image of a huge vegetation $(24 \times 12 \mathrm{~mm})$ attached to the anterior mitral leaflet. The diagnosis was confirmed by subsequent transesophageal echocardiography (TEE) examination that showed more precisely invagination of the vegetation into an MV orifice, which caused hemodynamically significant valve incompetence (Figs. $1 \mathrm{~A}, \mathrm{~B}$ ).

The patient was referred for emergency operative treatment after 2 weeks of advanced antibiotic therapy, which resulted in fever normalization prior the surgery. Transesophageal echocardiography performed in an operating room before the operation showed significant MV incompetence without leaflet prolapse and primary chordal disruption (type I - following Carpentier classification), which suggest leaflet(s) perforation. In addition, TEE revealed that the vegetation originated from the region of the P3 annulus, posterior leaflet and posteromedial commissure, while an empty annular abscess cavity was filled with mixed blood stream from both the atrial and ventricular side.

After hypothermic cardiopulmonary bypass institution with typical bicaval cannulation, the right atrium was opened and through an interatrial septum incision good exposure of the left atrium with the MV vegetation was achieved. The vegetation was carefully dissected with a margin of uninfected tissue showing a large P2/P3 perforation and an empty abscess cavity of the P3 annulus. Primary chordae were not disrupted. After local administration of Betadine a pericardial patch pretreated with $0.6 \%$ glutaraldehyde was sutured with single "U" monofilament 5-0 stitches reinforced by pericardial pledgets closing the defect in the P2/P3 posterior mitral leaflet (Fig. 2). The patch was implanted in such a manner as to close simultaneously the abscess cavity from the left atrial side and restore the posteromedial commissure. After intraoperative MV examination a paracommisural "edge-to-edge" A3/P3 repair was performed because of mild residual leak, while repeated saline controls showed no leaks and good leaflet coaptation. After weaning from cardio-pulmonary bypass subsequent TEE confirmed good MV competence (Fig. $1 \mathrm{C}$ ). There was some residual inflow to the empty abscess cavity from the ventricular side without communication to the left atrium (Fig. 1 D). This inflow was not further observed in follow-up TEE performed 3 months postoperatively. The postoperative course was uneventful. Antibiotic administration was continued up to 6 weeks due to therapeutic bacterial endocarditis treatment guidelines. Further transthoracic echocardiography examinations confirmed good valvular function after the repair. The patient was discharged home 6 weeks postoperatively with negative blood cultures and inflammatory parameters returned to the normal range (C-reactive protein - CRP, procalcitonin - PCT). Mid-term TTE examinations repeated 3, 6 and 12 months postoperatively showed a consistently competent MV. The boy returned to his normal life activity in NYHA class I.

Surgery in pediatric patients with acute endocarditis is still rare; thus there is still a limited amount of articles available concerning mitral valve repairs in children. There is no doubt that mitral valve repair is superior to replacement, especially in the subgroup of patients with expected

Address for correspondence: Jacek H. Juściński MD, PhD, Department of Cardiac Surgery, Medical University of Gdansk, 7 Dębinki St, 80-210 Gdansk, Poland, phone: +48 603611 954, e-mail: jackju@gumed.edu.pl

Received: 6.02.2018, accepted: 17.04.2018. 

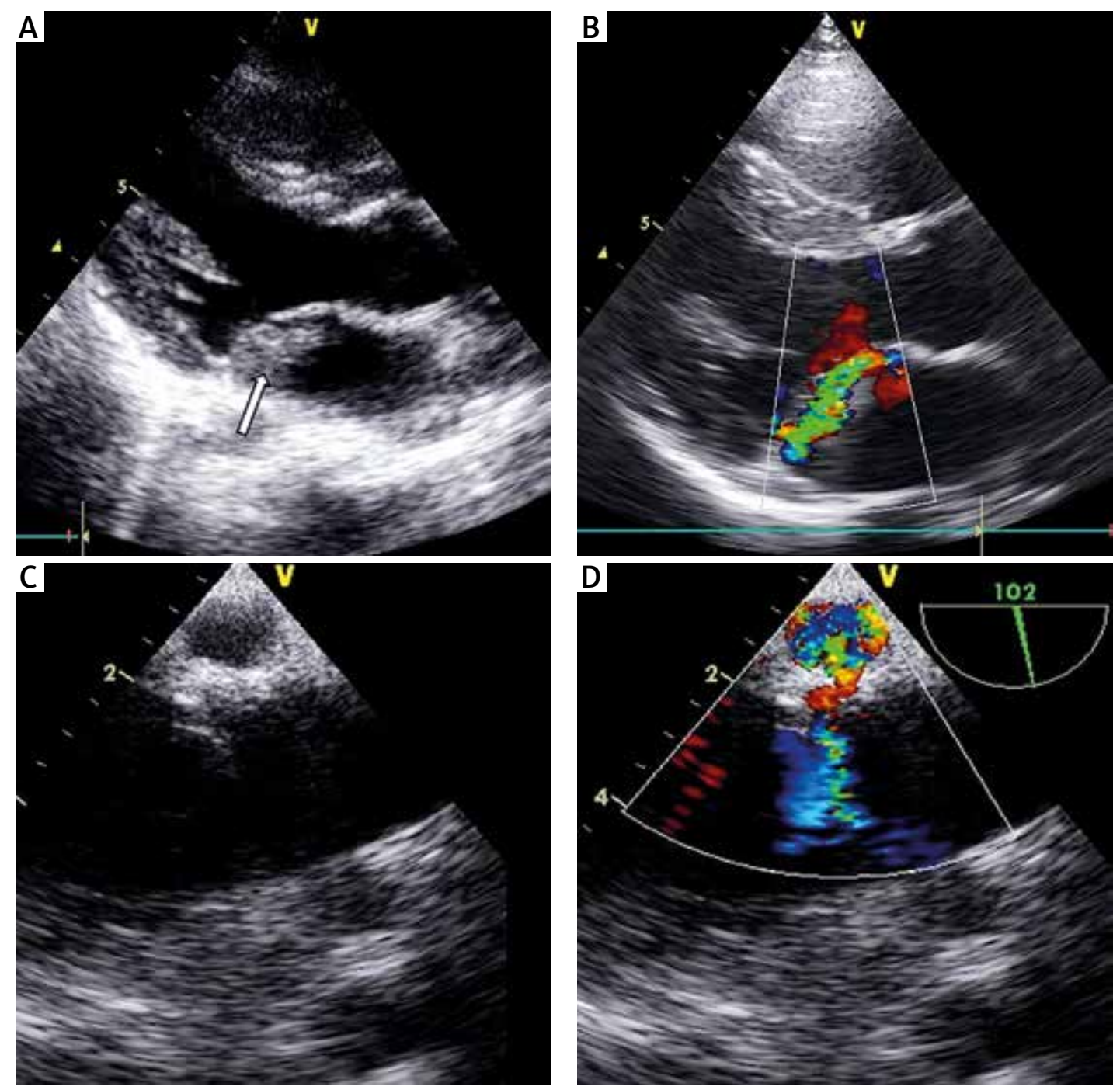

Fig. 1. A - Transthoracic echocardiography: image of huge bacterial vegetation in left atrium (arrow). B - Transthoracic echocardiography: image of preoperative mitral valve incompetence. C - Transesophageal echocardiography: image directly after reconstruction in operating room, competent mitral valve, $\mathbf{D}$ - residual inflow into empty abscess cavity from ventricular site

long-life survival, growth potential and contraindications to anticoagulation [1, 2].

With regard to surgical strategy, all infected tissues need to be carefully excised before valve reconstruction with a pericardial patch. Ushijima et al. [3] described successful MV repair performed while almost half of a valve was removed and pericardial reconstruction covered the A3/P3 segments with the posteromedial commissure. They fixed the patch to a twisted pericardial strip incorporated into the destroyed annulus region. Similar reconstruction was presented in the report of Ishimaru et al. [4], although his team preferred pericardial patch suturing to a left atrial wall in this region. Both cited authors used the continuous suture technique for patch implantation. In our case, in the settings of bacterial endocarditis, single " $U$ " monofilament stitches reinforced with pericardial pledgets were the method of choice. This technique provided better safety and stability for the implanted patch in the bacterial environment, and allowed for optimal blood supply in the re- gion of the pericardial patch suture line. We believe that this reconstruction enables optimal penetration of antibiotics [5].

Our patient required P2/P3 reconstruction including posteromedial commissure. For better leaflet coaptation and residual leak elimination we add paracommisural "edge-to-edge" A3/P3 sutures. In our opinion, if there is no primary chordae rupture repair, this maneuver is possible in every pediatric patient with bacterial endocarditis involving the MV, despite very low body weight. Healy et al. [6] described successful repair of an anterior mitral leaflet in a $1.9 \mathrm{~kg}$ infant with endocarditis, and stressed that retention of primary chordae was crucial for a successful operation. We correspond with the authors, who earlier concluded that $M V$ reconstruction, if only technically possible, should be the therapy of choice in the case of bacterial endocarditis, particularly in children and adolescents $[2,6]$. Mid-term results presented by Takahashi et al. [7] also confirm these observations. 
Our goal was to restore mitral valve function and, whenever possible, its anatomy - as concluded in all discussed papers. In comparison to previously presented repair techniques performed in non-infected tissues, our intention was to avoid the use of any artificial material, such as non-biodegradable rings, artificial chords, or pledgets $[8,9]$. The only exception from artificial prosthetic materials use, planned before the surgery, was primary chordal rupture to the anterior mitral leaflet, when Gore-Tex chordae would became necessary for sufficient repair.

Pediatric mitral valve repair avoiding artificial materials was found to be superior to valve replacement, especially in a pediatric patient with expected long-life survival, growth potential and natural contraindications to anticoagulation.

An acute period of mitral valve bacterial endocarditis was not a contraindication for primary repair.

\section{Disclosure}

Authors report no conflict of interest.

\section{References}

1. Haponiuk I, Mozol K, Gierat-Haponiuk K, Kansy A, Burczyński P, Maruszewski B. Profilaktyka powikłań zakrzepowo-zatorowych po paliatywnych operacjach kardiochirurgicznych u dzieci. SMP 2007; 9: 70-74.

2. Haponiuk I, Chojnicki M, Jaworski R, Steffens M, Paczkowski K, Szofer-Sendrowska A, Paśko-Majewska M, Gierat-Haponiuk K, Romanowicz A, Szymanowicz W. Pediatric Melody mitral valve replacement in acute endocarditis - alternative surgical-hybrid technique. Kardiol Pol 2017; 75: 845-849.

3. Ushijima T, Kikuchi Y, Takato M, Yamamoto Y, Kawachi K, Watanabe G. Commissural autologous pericardial patch repair: a novel technique for active mitral valve endocarditis involving the mitral annulus. Ann Thorac Surg 2009; 88: 29-30.

4. Ishimaru K, Nishigaki K, Kanaya T, Araki K, Shibata T. Modified commissural patch repair in a child with active mitral endocarditis. Asian Cardiovasc Thorac Ann 2016; 24: 45-47.

5. Yamaguchi H, Eishi K. Surgical treatment of active infective mitral valve endocarditis. Ann Thorac Cardiovasc Surg 2007; 13: 150-155.

6. Healy DG, Wood AE. Anterior mitral leaflet reconstruction with pericardium in a 1.9 kg infant with endocarditis. Ann Thorac Surg 2006; 81: 2310-2312.

7. Takahashi H, Kadowaki T, Maruo A, Yutaka O, Oshima Y. Mid-term results of mitral valve repair with autologous pericardium in pediatric patients. J Heart Valve Dis 2014; 23: 302-309.

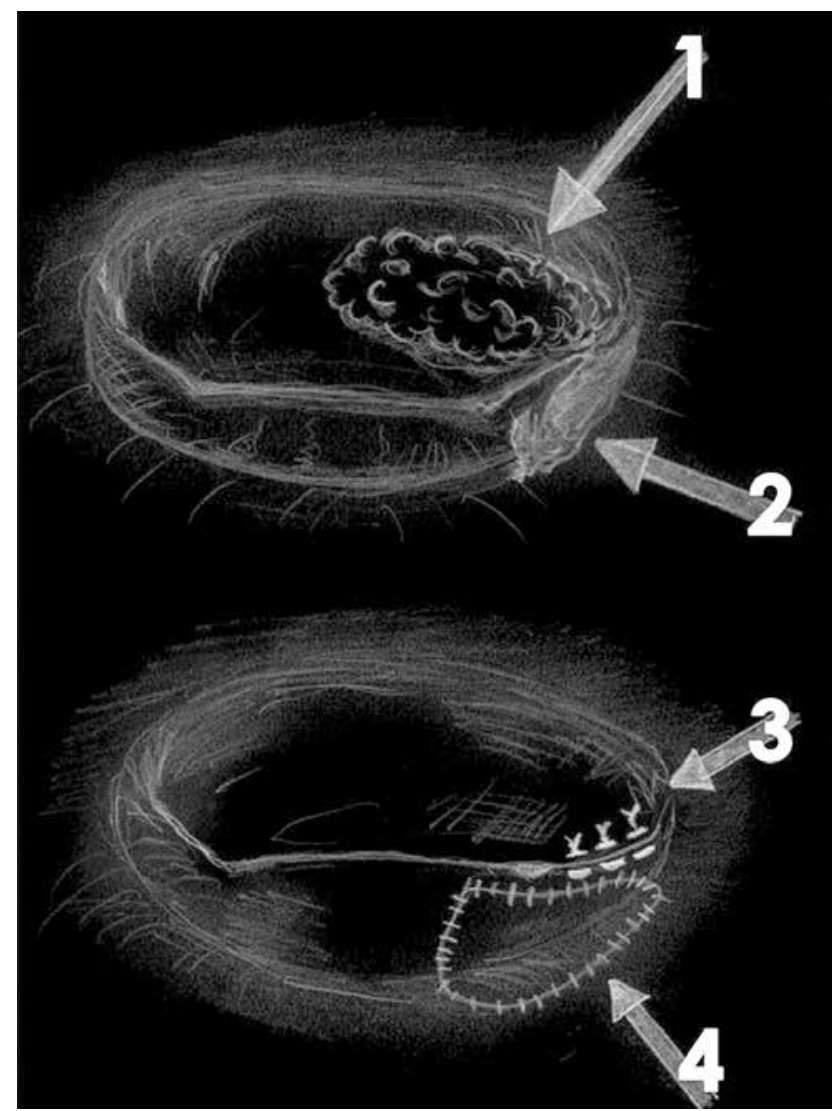

Fig. 2. Operative technique scheme - arrows: 1 - vegetation, 2 abscess cavity of P3 annulus, 3 - paracommisural "edge-to-edge" $\mathrm{A} 3 / \mathrm{P} 3$ repair, 4 - pericardial patch closing the defect in P2/P3 posterior mitral leaflet

8. Perrotta S, Zubrytska Y. Valve selection in aortic valve endocarditis. Kardiochir Torakochir Pol 2016; 13: 203-209.

9. Wojtalik M, Mrówczyński W, Mrozinski B. Valve-sparing surgery for isolated cleft mitral valve complicated by endocarditis in a child. J Heart Valve Dis 2011; 20: 98-99. 